\title{
An Approach for Synthesis of Modulated M-Channel FIR Filter Banks Utilizing the Frequency-Response Masking Technique
}

\author{
Linnéa Rosenbaum, Per Löwenborg, and Håkan Johansson \\ Department of Electrical Engineering, Linköping University, 58183 Linköping, Sweden
}

Received 22 December 2005; Revised 29 June 2006; Accepted 26 August 2006

Recommended by Soontorn Oraintara

\begin{abstract}
The frequency-response masking (FRM) technique was introduced as a means of generating linear-phase FIR filters with narrow transition band and low arithmetic complexity. This paper proposes an approach for synthesizing modulated maximally decimated FIR filter banks (FBs) utilizing the FRM technique. A new tailored class of FRM filters is introduced and used for synthesizing nonlinear-phase analysis and synthesis filters. Each of the analysis and synthesis FBs is realized with the aid of only three subfilters, one cosine-modulation block, and one sine-modulation block. The overall FB is a near-perfect reconstruction (NPR) FB which in this case means that the distortion function has a linear-phase response but small magnitude errors. Small aliasing errors are also introduced by the FB. However, by allowing these small errors (that can be made arbitrarily small), the arithmetic complexity can be reduced. Compared to conventional cosine-modulated FBs, the proposed ones lower significantly the overall arithmetic complexity at the expense of a slightly increased overall FB delay in applications requiring narrow transition bands. Compared to other proposals that also combine cosine-modulated FBs with the FRM technique, the arithmetic complexity can typically be reduced by $40 \%$ in specifications with narrow transition bands. Finally, a general design procedure is given for the proposed FBs and examples are included to illustrate their benefits.
\end{abstract}

Copyright (c) 2007 Linnéa Rosenbaum et al. This is an open access article distributed under the Creative Commons Attribution License, which permits unrestricted use, distribution, and reproduction in any medium, provided the original work is properly cited.

\section{INTRODUCTION}

Maximally decimated FBs (see Figure 1) find applications in numerous areas [1-3]. Over the past two decades, a vast number of papers on the theory and design of such FBs have been published. Traditionally, the attention has to a large extent been paid to the problem of designing perfect reconstruction (PR) FBs. In a PR FB, the output sequence of the overall system is simply a shifted version of the input sequence. However, FBs are most often used in applications where small errors (emanating from quantizations, etc.) are inevitable and allowed. Imposing PR on the FB is then an unnecessarily severe restriction which may lead to a higher arithmetic complexity than is actually required to meet the specification at hand (arithmetic complexity is defined in this article as the number of arithmetic operations per sample needed in an implementation of an FB). To reduce the complexity one should therefore use near perfect reconstruction (NPR) FBs. For example, it is demonstrated in [4-6] that the complexity can be reduced significantly by using NPR instead of PR FBs. For this reason, this paper proposes a new class of FBs with nearly perfect reconstruction. The distortion function has a linear-phase response but a small magnitude distortion. Further, small aliasing errors are present. The magnitude distortion and aliasing errors can however be made arbitrarily small by properly designing a prototype filter, and a general design procedure for this purpose is presented. Compared to conventional cosine modulated FBs as well as similar approaches, the proposed ones lower the overall arithmetic complexity significantly, in applications requiring narrow transition bands. An example of such an application is frequency-band decomposition for parallel sigmadelta systems [7] (what is gained using parallelism, is lost with a wide transition band). In the former comparison, also the number of distinct coefficients is reduced significantly, at the expense of a slightly increased overall delay. Apart from the NPR property, the main features of the FBs presented here are the following.

\section{Modulation}

Regular cosine modulated FBs are widely used and known to be highly efficient, since each of the analysis and synthesis 


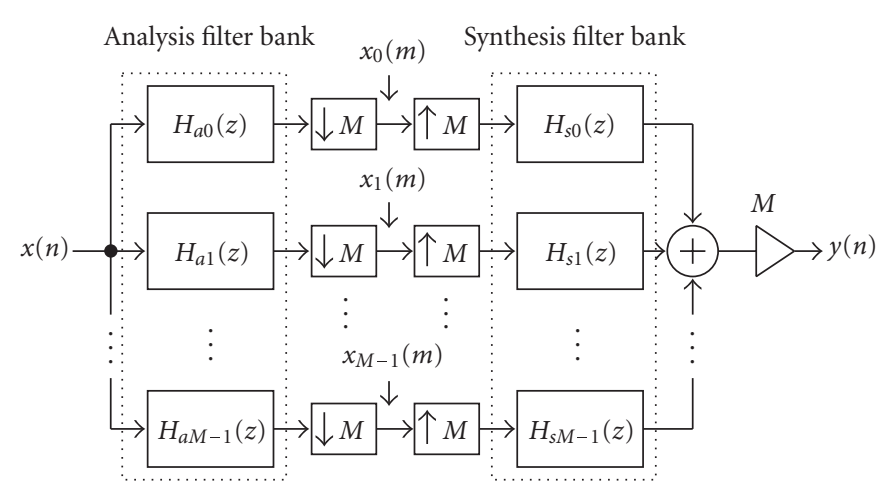

FIgURE 1: $M$-channel maximally decimated FB.

parts can be implemented with the aid of only one (prototype) filter and a discrete cosine transform [2]. The efficiency of this technique is exploited in the article after appropriate modifications. Specifically, both cosine and sine modulations are utilized together with a modified class of FRM filters (see below), which generates efficient overall FBs.

\section{Frequency-response masking (FRM)}

When the transition bands of the filters are narrow, the overall complexity may be high. This is due to the fact that the order of an FIR filter is inversely proportional to the transition bandwidth [8]. To alleviate this problem, one can use the FRM technique which was introduced as a means of generating linear-phase FIR filters with both narrow transition band and low arithmetic complexity [9-12]. However, to make the technique suitable for the proposed modulated FBs, we introduce a modified class of FRM filters. This modified class has been considered in $[13,14]$, but not in the context of $M$-channel FBs. The main difference is that these FRM filters have a nonlinear-phase response whereas the traditional ones have a linear-phase response. The proposed FRM filters are used as prototype filters in the proposed cosine and sine modulation-based FBs. Each of the analysis and synthesis FBs is realized with the aid of three subfilters, one cosine modulation block, and one sine modulation block. The reason for using the modified FRM filters in the proposed modulation scheme is that the corresponding FB structure requires a lower arithmetic complexity. Using instead the conventional FRM filters, one would need three cosine modulation blocks.

\section{Few optimization parameters}

Another advantage of the proposed FB class is that the number of parameters to optimize is few, which is an important issue in extensive designs. Efficient structures are given for implementing the proposed FBs, and procedures for optimizing them in the minimax sense are described.

\section{Relation to previous work}

Cosine modulated FIR FBs based on the original FRM filters have been considered in [15-19]. The resulting structure requires only one modulation block in each of the analysis and synthesis parts but, on the other hand, additional upsamplers (and downsamplers) are needed, which makes some subfilters work at an unnecessarily high sampling rate. The focus is also different, since the goal in [15-19] is to minimize the number of optimization parameters and not the arithmetic complexity. It should also be noted that, except for two examples in $[18,19]$, the examples in $[15-$ 19] have filter specifications where only one branch in the FRM structure is needed. For such specifications, the arithmetic complexity is not lower than for that of a regular direct-form FIR prototype filter. Thus, in terms of multiplications per input/output sample there is nothing to gain using narrow-band (one-branch) FRM prototype filters, and therefore they are not discussed in this paper. Finally, it is noted that this paper is an extension of the work presented at two conferences $[20,21]$, where the basic principles were introduced without giving all details presented in this paper.

The outline of the paper is as follows: in Section 2, a brief treatment of the conventional FRM technique is given. After that, the proposed FB is described in detail in Section 3. This section also includes some important properties and a realization of the FB class. Section 4 gives a general design procedure, followed by a design example and comparisons in Section 5. The paper is concluded in Section 6.

\section{FRM TECHNIQUE}

As an introduction to FRM, the conventional FRM technique for generating lowpass linear-phase filters is reviewed in this section. The modifications used in the proposed FB class are described in the subsequent section.

In the frequency-response masking technique, the transfer function of the overall filter is expressed as [9-12]

$$
H(z)=G\left(z^{L}\right) F_{0}(z)+G_{c}\left(z^{L}\right) F_{1}(z)
$$




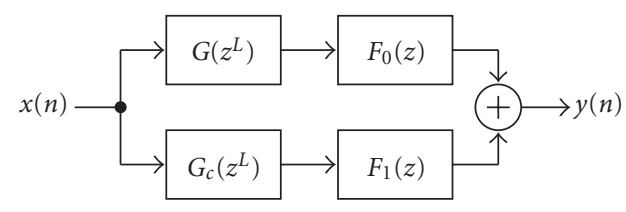

FIgURE 2: Structure used in the FRM approach.

where $G(z)$ and $G_{c}(z)$ are referred to as the model filter and complementary model filter, respectively. The filters $F_{0}(z)$ and $F_{1}(z)$ are referred to as the masking filters which extract one or several passbands of the periodic model filter $G\left(z^{L}\right)$ and periodic complementary ${ }^{1}$ model filter $G_{c}\left(z^{L}\right)$. The structure is illustrated in Figure 2 and typical magnitude responses of the subfilters as well as the resulting filter can be seen in Figure 3 in the next section.

The FRM technique was originally introduced in [10] as a means to reduce the arithmetic complexity of linear-phase FIR filters with narrow transition bands. In this approach, $G(z)$ and $G_{c}(z)$ have to be even-order linear-phase filters of equal delays and form a complementary filter pair, whereas both $F_{0}(z)$ and $F_{1}(z)$ are either even- or odd-order linearphase filters of equal delays. These filters could be used directly to generate the analysis and synthesis filters in the proposed modulated FB scheme to be considered in the following section, but the result is that each of the analysis and synthesis FB then requires three modulation blocks. Therefore, we introduce in the next section modified FRM FIR filters that make it possible to use only two modulation blocks. These modified FRM FIR filters have been considered in $[13,14]$ but not in the context of $M$-channel FBs.

\section{PROPOSED FILTER BANKS}

This section gives transfer functions, properties, and realizations of the proposed FBs. The choices of prototype filters and analysis and synthesis transfer functions assure the overall filter bank to fulfill the NPR criteria.

\subsection{Prototype filter transfer functions}

For the proposed modulated FBs, the transfer functions of the analysis and synthesis filters are generated from the prototype filter transfer functions $P_{a}(z)$ and $P_{s}(z)$, respectively. These transfer functions are given by

$$
\begin{aligned}
& P_{a}(z)=G\left(z^{L}\right) F_{0}(z)+G_{c}\left(z^{L}\right) F_{1}(z), \\
& P_{s}(z)=G\left(z^{L}\right) F_{0}(z)-G_{c}\left(z^{L}\right) F_{1}(z) .
\end{aligned}
$$

Typical magnitude responses for the model filter, the masking filter, and overall filter $P_{a}(z)$ are as shown in Figure 3 . The transition band of $P_{a}(z)$ (and $P_{s}(z)$ ) can be selected to

\footnotetext{
${ }^{1}$ In the case of linear-phase FIR filters, this means that the sum of the zerophase frequency responses of the filter pair is equal to unity.
}

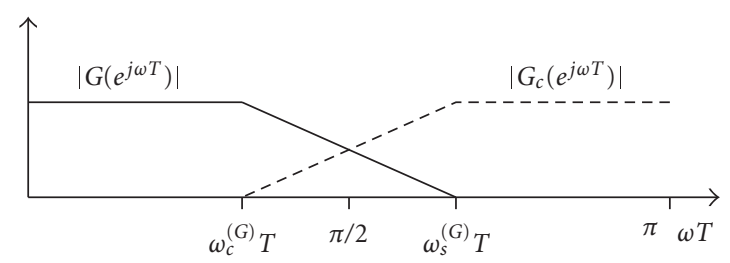

(a)

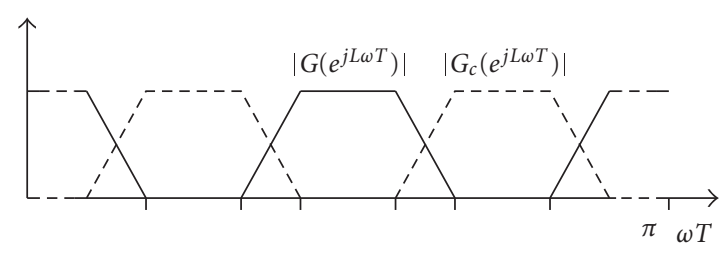

(b)

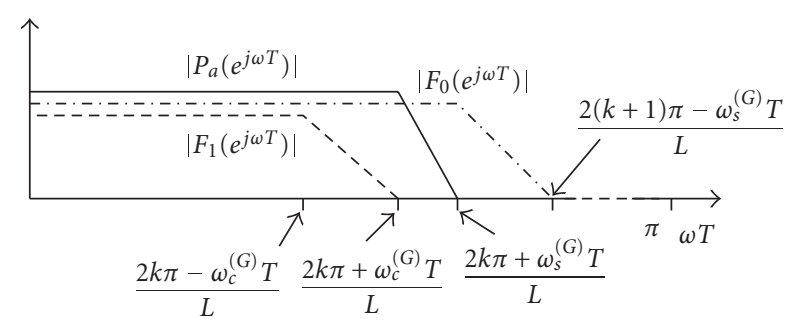

(c)

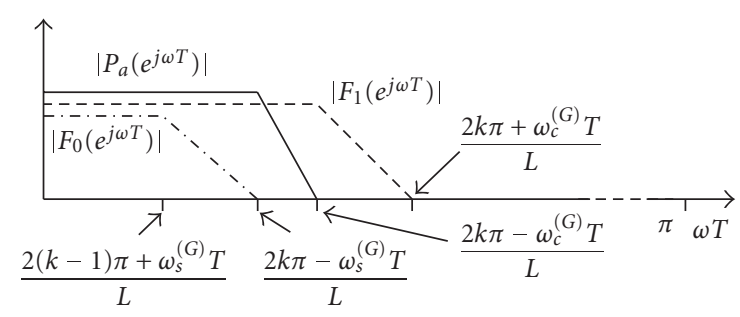

(d)

FIGURE 3: Illustration of magnitude functions in the FRM approach, where (c) and (d) show the two alternatives Case 1 and Case 2, respectively.

be one of the transition bands provided by either $G\left(z^{L}\right)$ or $G_{c}\left(z^{L}\right)$. We refer to these two different cases as Case 1 and Case 2, respectively. Further, we let $\omega_{c} T, \omega_{s} T, \delta_{c}$, and $\delta_{s}$ denote the passband edge, stopband edge, passband ripple, and stopband ripple, respectively, for the overall filter $P_{a}(z)$ (and $\left.P_{s}(z)\right)$. For the model and masking filters $G(z), G_{c}(z), F_{0}(z)$, and $F_{1}(z)$, additional superscripts $(G),\left(G_{c}\right),\left(F_{0}\right)$, and $\left(F_{1}\right)$, respectively, are included in the corresponding ripples and edges. The periodicity $L$, and the subfilters $G(z), G_{c}(z), F_{0}(z)$, and $F_{1}(z)$ are selected to satisfy the following criteria.

(i) The model filters $G(z)$ and $G_{c}(z)$ are linear-phase FIR filters of odd order $N_{G}$, with symmetrical and antisymmetrical impulse responses, respectively. They are related as

$$
G_{c}(z)=G(-z)
$$


and designed to be approximately power complementary (i.e., $\left.\left|G\left(e^{j \omega T}\right)\right|^{2}+\left|G_{c}\left(e^{j \omega T}\right)\right|^{2} \approx 1\right)$. This is mainly what distinguishes the proposed FRM filters from the conventional ones, ${ }^{2}$ and it means for example that the transition band of $G(z)$ must be centered at $\pi / 2$.

(ii) $L$ is an integer related to the number of channels $M$ as

$$
L= \begin{cases}(4 m+1) M, & \text { Case } 1, \\ (4 m-1) M, & \text { Case } 2 .\end{cases}
$$

The reason for this restriction is that the transition band of the FRM filter (see the illustration of the two different cases in Figures 3(c) and 3(d)) must coincide with the transition band of the prototype filter at $\pi / 2 M$. Thus,

$$
\frac{2 k \pi \pm \pi / 2}{L}=\frac{\pi}{2 M} .
$$

(iii) The masking filters $F_{0}(z)$ and $F_{1}(z)$ are of order $N_{F}$ and linear-phase lowpass filters with symmetrical impulse responses. The filter order can be either even or odd. Further, in order to ensure approximate power complementarity of the analysis filters, additional restrictions in the transition bands of $P_{a}(z)$ and $P_{s}(z)$ must be added. This leads to slightly tightened restrictions on the passband and stopband edges of the masking filters compared to [10], which is illustrated in Figure 3.

\subsection{Analysis and synthesis filter transfer functions}

For Case 1, the analysis filters $H_{a k}(z)$ and synthesis filters $H_{s k}(z)$ are obtained by modulating the prototype filters $P_{a}(z)$ and $P_{s}(z)$ according to

$$
\begin{gathered}
H_{a k}(z)=\beta_{k} P_{a}\left(z W_{2 M}^{(k+0.5)}\right)+\beta_{k}^{*} P_{a}\left(z W_{2 M}^{-(k+0.5)}\right), \\
H_{s k}(z)=c j(-1)^{k}\left[\beta_{k} P_{s}\left(z W_{2 M}^{(k+0.5)}\right)-\beta_{k}^{*} P_{s}\left(z W_{2 M}^{-(k+0.5)}\right)\right],
\end{gathered}
$$

respectively, for $k=0,1, \ldots, M-1$, with

$$
c= \begin{cases}-1, & N_{G}+1=4 m, \\ 1, & N_{G}+1=4 m+2\end{cases}
$$

for some integer $m$, and

$$
W_{M}=e^{-j 2 \pi / M}, \quad \beta_{k}=w_{2 M}^{(k+0.5) N_{F} / 2} .
$$

For Case 2, (9) is negated. Note that this type of modulation is slightly different from the one that is usually employed in cosine-modulated FBs [2]. For example, $\theta_{k}$ in [2]

\footnotetext{
${ }^{2}$ For the conventional FRM filters, $N_{G}$ must be even and $G_{c}(z)=z^{-N_{G} / 2}$ $G(z)$. In this case, it is not possible to make $G(z)$ and $G_{c}(z)$ approximately power complementary.
}

is not needed here, since power complementarity can be achieved directly by choosing the model filters according to Section 3.1. The main difference is though that unlike the conventional ones, the proposed prototype filters have a nonlinear-phase response. Nevertheless, by the choices in (7)-(10), the FB is ensured to have all the important properties that are stated later in Section 3.3.

\subsection{Filter bank properties}

This section gives five important properties of the proposed FBs useful in the design procedure. Proofs of the first four properties are given in the appendix. The fifth property is shown in Section 4.

(1) The magnitude responses of $P_{a}(z)$ and $P_{s}(z)$ are equal, that is,

$$
\left|P_{a}\left(e^{j \omega T}\right)\right|=\left|P_{s}\left(e^{j \omega T}\right)\right|
$$

(2) The cascaded filter $P_{a}(z) P_{s}(z)$ has a linear-phase response.

(3) The magnitude responses of $H_{a k}(z)$ and $H_{s k}(z)$ are equal, that is,

$$
\left|H_{a k}\left(e^{j \omega T}\right)\right|=\left|H_{s k}\left(e^{j \omega T}\right)\right| .
$$

(4) The distortion transfer function $V_{0}(z)$ (see Section 4) has a linear-phase response with a delay of $L N_{G}+N_{F}$ samples.

(5) The FBs can readily be designed in such a way that (a) the analysis and synthesis filters are arbitrarily good frequency-selective filters, and (b) the magnitude distortion and aliasing errors are arbitrarily small.

\subsection{Filter bank structures}

In this section it is shown how to realize the proposed analysis FB class with two modulation blocks instead of three. The synthesis FB can be realized in a corresponding way [2]. We begin by expressing $G(z)$ and $G_{c}(z)$ in polyphase forms according to

$$
\begin{gathered}
G(z)=G_{0}\left(z^{2}\right)+z^{-1} G_{1}\left(z^{2}\right), \\
G_{c}(z)=G(-z)=G_{0}\left(z^{2}\right)-z^{-1} G_{1}\left(z^{2}\right)
\end{gathered}
$$

so that $P_{a}(z)$ in $(2)$ can be written on the form

$$
P_{a}(z)=G_{0}\left(z^{2 L}\right) A(z)+z^{-L} G_{1}\left(z^{2 L}\right) B(z) .
$$

In (14), the filters $A(z)$ and $B(z)$ are the sum and the difference of the two masking filters according to

$$
A(z)=F_{0}(z)+F_{1}(z), \quad B(z)=F_{0}(z)-F_{1}(z) .
$$


The analysis filters $H_{a k}(z)$ can then be written as

$$
H_{a k}(z)=G_{0}\left(-z^{2 L}\right) A_{k}(z)+s(-1)^{k} j z^{-L} G_{1}\left(-z^{2 L}\right) B_{k}(z),
$$

where

$$
\begin{gathered}
A_{k}(z)=\beta_{k} A\left(z W_{2 M}^{(k+0.5)}\right)+\beta_{k}^{*} A\left(z W_{2 M}^{-(k+0.5)}\right), \\
B_{k}(z)=\beta_{k} B\left(z W_{2 M}^{(k+0.5)}\right)-\beta_{k}^{*} B\left(z W_{2 M}^{-(k+0.5)}\right), \\
\quad s= \begin{cases}-1, & \text { Case } 1, \\
1, & \text { Case } 2 .\end{cases}
\end{gathered}
$$

As seen in (16), $G_{0}\left(-z^{2 L}\right)$ and $G_{1}\left(-z^{2 L}\right)$ are conveniently independent of $k$ and are thus the same in each channel.

Let $a(n), b(n), a_{k}(n)$, and $b_{k}(n)$ denote the impulse responses of $A(z), B(z), A_{k}(z)$, and $B_{k}(z)$, respectively. We then get from (17) and (10) that $a_{k}(n)$ and $b_{k}(n)$ are related to $a(n)$ and $b(n)$ through

$$
\begin{aligned}
& a_{k}(n)=2 a(n) \cos \left(\frac{(2 k+1) \pi}{2 M}\left(n-\frac{N_{F}}{2}\right)\right), \\
& b_{k}(n)=2 j b(n) \sin \left(\frac{(2 k+1) \pi}{2 M}\left(n-\frac{N_{F}}{2}\right)\right) .
\end{aligned}
$$

Since $b_{k}(n)$ is purely imaginary, $H_{a k}(z)$ is obviously the transfer function of a filter with a real impulse response. It can be written as

$$
H_{a k}(z)=G_{0}\left(-z^{2 L}\right) A_{k}(z)-s(-1)^{k} z^{-L} G_{1}\left(-z^{2 L}\right) B_{k R}(z),
$$

where

$$
B_{k R}(z)=-j B_{k}(z)
$$

Through a similar derivation as above, the synthesis filters $H_{s k}(z)$ can be rewritten as

$$
H_{s k}(z)=(-1)^{k} G_{0}\left(-z^{2 L}\right) B_{k R}(z)+s z^{-L} G_{1}\left(-z^{2 L}\right) A_{k}(z) .
$$

The realization of the analysis FB is shown in Figure 4, where $Q_{i}^{(A)}\left(-z^{2}\right)$ and $Q_{i}^{(B)}\left(-z^{2}\right), i=0,1, \ldots, 2 M-1$, are the polyphase components of $A(z)$ and $B(z)$, respectively. The cosine modulation block $\mathbf{T}_{1}$ is a simplified version of the corresponding one in [2] (with $\theta_{k}=0$ ). It consists of two trivial matrices and an $M \times M$ DCT-IV matrix. The other one, $\mathrm{T}_{2}$, is a corresponding sine modulation block. Further, because of symmetry in the coefficients of $G(z)$, the two filters $G_{0}\left(-z^{2}\right)$ and $G_{1}\left(-z^{2}\right)$ can share multipliers. This is illustrated for the 0 th channel and filter order $N_{G}=3$, in Figure 5. Although we have three subfilters to implement, $G(z), F_{0}(z)$, and $F_{1}(z)$, we have been able to reduce the number of modulation blocks needed from three to only two.

\section{FILTER BANK DESIGN}

For $M$-channel maximally decimated FBs (see Figure 1 ) the $z$-transform of the output signal is given by

$$
Y(z)=\sum_{m=0}^{M-1} V_{m}(z) X\left(z W_{M}^{m}\right),
$$

where

$$
V_{m}(z)=\sum_{k=0}^{M-1} H_{a k}\left(z W_{M}^{m}\right) H_{s k}(z) .
$$

Here, $V_{0}(z)$ is the distortion transfer function whereas the remaining $V_{m}(z)$ are the aliasing transfer functions. For a PR (near-PR) FB, it is required that the distortion function is (approximates) a delay, and that the aliasing components are (approximate) zero. We now derive expressions for the specification of the model filter $G(z)$ and the masking filters $F_{0}(z)$ and $F_{1}(z)$, in order for the analysis filters $H_{a k}(z)$, the distortion function $V_{0}(z)$, and the aliasing terms $V_{m}(z)$, to fulfill a given specification.

Let the specifications of $H_{a k}(z)$ be

$$
\begin{gathered}
1-\delta_{c} \leq\left|H_{a k}\left(e^{j \omega T}\right)\right| \leq 1+\delta_{c}, \quad \omega T \in \Omega_{c, k}, \\
\left|H_{a k}\left(e^{j \omega T}\right)\right| \leq \delta_{s}, \quad \omega T \in \Omega_{s, k}
\end{gathered}
$$

where $\Omega_{c, k}$ and $\Omega_{s, k}$, respectively, are the passband and stopband regions of $H_{k}(z)$. Expressed with the aid of $\Delta$, where $\Delta$ is half the transition bandwidth, they are as illustrated in Figure 6. Furthermore, the magnitude of the distortion and aliasing functions are to meet

$$
\begin{gathered}
1-\delta_{0} \leq\left|V_{0}\left(e^{j \omega T}\right)\right| \leq 1+\delta_{0}, \quad \omega T \in[0, \pi], \\
\left|V_{m}\left(e^{j \omega T}\right)\right| \leq \delta_{1}, \quad \omega T \in[0, \pi], m=0,1, \ldots, M-1,
\end{gathered}
$$

respectively. To fulfill the above specifications, the following optimization problem is solved:

minimize $\delta$

$$
\begin{aligned}
& \text { subject to }|| H_{a k}\left(e^{j \omega T}\right)|-1| \leq \delta\left(\frac{\delta_{c}}{\delta_{1}}\right), \quad \omega T \in \Omega_{c, k}, \\
& \qquad\left|H_{a k}\left(e^{j \omega T}\right)\right| \leq \delta\left(\frac{\delta_{s}}{\delta_{1}}\right), \quad \omega T \in \Omega_{s, k}, \\
& || V_{0}\left(e^{j \omega T}\right)|-1| \leq \delta\left(\frac{\delta_{0}}{\delta_{1}}\right), \quad \omega T \in[0, \pi], \\
& \left|V_{m}\left(e^{j \omega T}\right)\right| \leq \delta, \quad \omega T \in[0, \pi] .
\end{aligned}
$$

The adjustable parameters in (28) are the filter coefficients of the subfilters $G(z), F_{0}(z)$, and $F_{1}(z)$, and $\delta$. For the specifications (25)-(27) to be fulfilled, we must find a solution with $\delta \leq \delta_{1}$. The problem is a nonlinear optimization problem and therefore requires a good initial solution. For this purpose, we first optimize $G(z), F_{0}(z)$, and $F_{1}(z)$ separately 


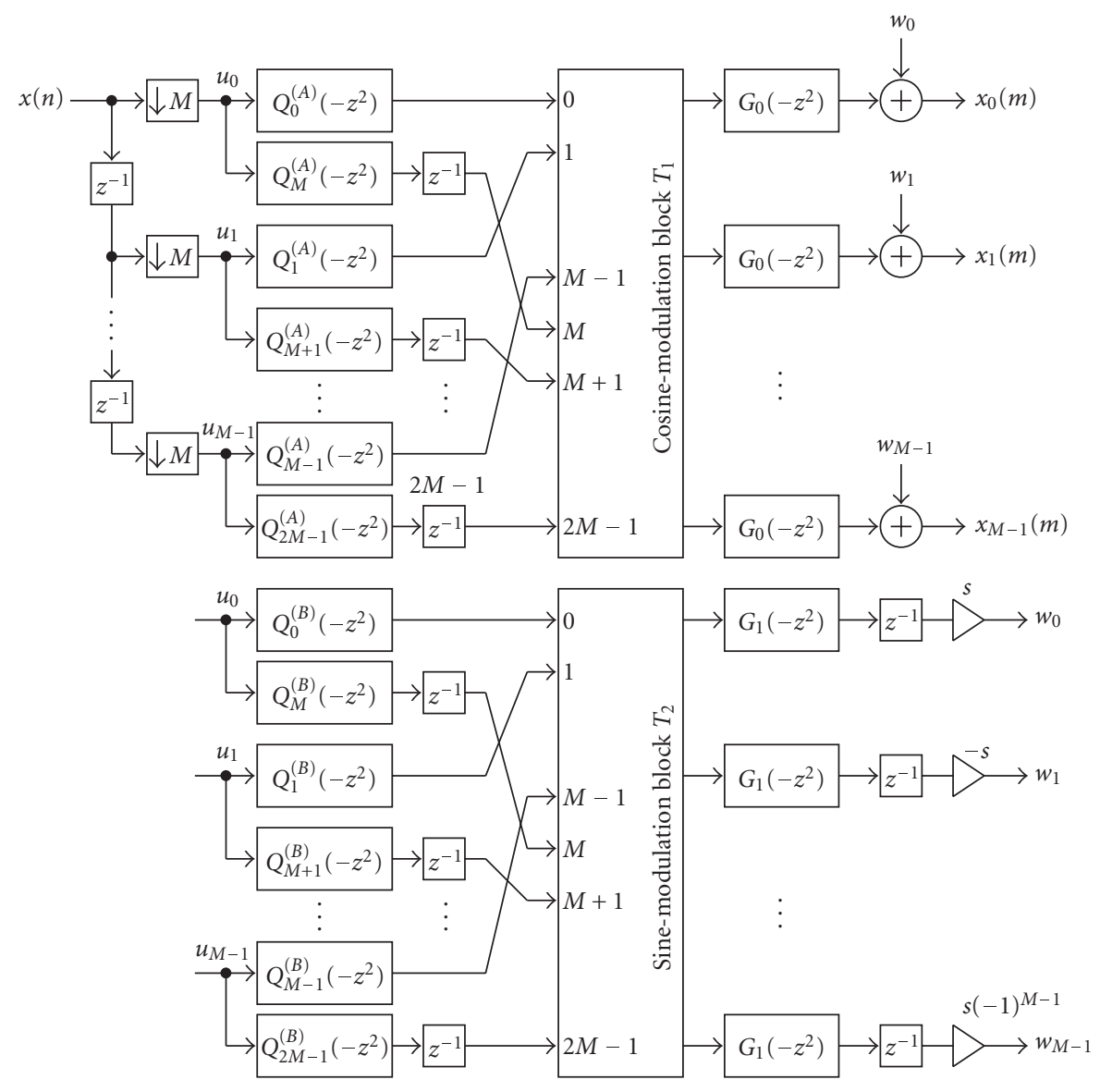

FIgURE 4: Realization of the proposed analysis FB.

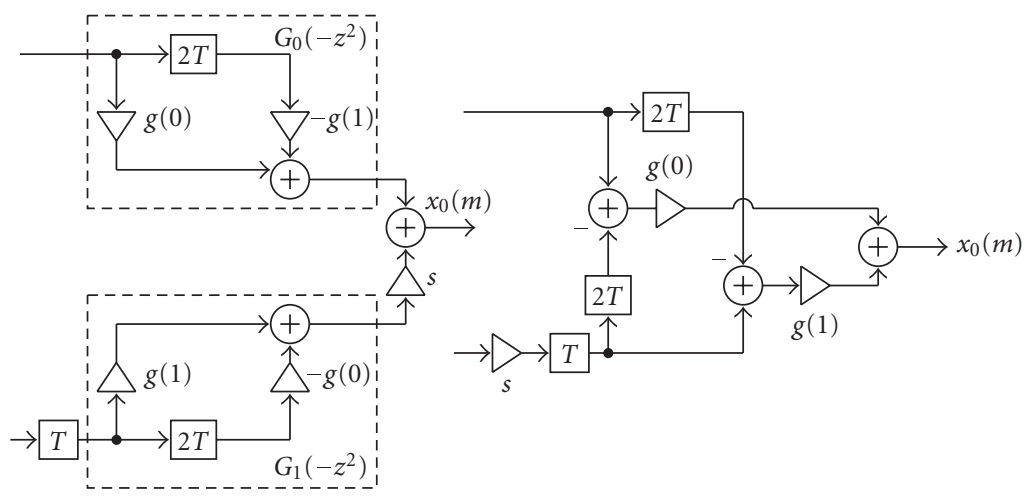

Figure 5: Sharing of multipliers between $G_{0}\left(-z^{2}\right)$ and $G_{1}\left(-z^{2}\right)$ in the 0 th channel when $N_{G}=3$.

and then these filters can serve as a good initial solution for further optimization according to (28).

In the following three sections, we give formulas for designing $G(z), F_{0}(z)$, and $F_{1}(z)$, so that they together fulfill a general specification of an NPR FB. These formulas are based on worst-case assumptions, and therefore in general, we get some unnecessary design margin. Because of this, it might be possible to successively decrease the filter orders of the subfilters and still satisfy the given specifications (25)-(27) after simultaneous optimization.

For some specifications, for example, when $M$ is large, it might not be possible to do simultaneous optimization. Then, separate optimization can be used exclusively and give a good (although not optimal) solution. The masking filters 


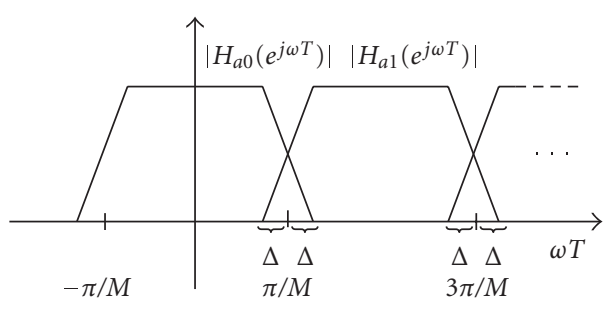

Figure 6: Passband and stopband regions for $H\left(e^{j \omega T}\right)$.

$F_{0}(z)$ and $F_{1}(z)$ can be designed using McClellan-Parks algorithm [22] or linear programming to fulfill $\delta_{c}^{\left(F_{0}\right)}, \delta_{s}^{\left(F_{0}\right)}$, and $\delta_{c}^{\left(F_{1}\right)}, \delta_{s}^{\left(F_{1}\right)}$, respectively. The model filter $G(z)$ should be designed to fulfill $\delta_{c}^{(G)}$ and $\delta_{s}^{(G)}$ but also to be approximately power complementary with a maximally allowed error of $\delta_{P C}$. To this end, nonlinear optimization must be used, and, for example, the algorithm in [22] can be used as a initial solution. Throughout the paper, the nonlinear optimization is performed in the minimax sense, but optimization in, for example, the least square sense is also possible after minor modifications. ${ }^{3}$

\subsection{Analysis filters}

In order to fulfill the specification of frequency selectivity of the analysis filters, the magnitude of $H_{a k}(z)$ is studied, as a function of the three subfilters $G(z), F_{0}(z)$, and $F_{1}(z)$. For convenience, we use the notation $X^{( \pm k)}(z)$ which stands for

$$
X\left(e^{ \pm((2 k+1) / 2 M) \pi} z\right) .
$$

This notation allows the transfer functions of the analysis filters to be written on the form

$$
H_{a k}(z)=G^{(-k)}\left(z^{L}\right) E_{0 k}(z)+G_{c}^{(-k)}\left(z^{L}\right) E_{1 k}(z),
$$

where $E_{0 k}(z)$ and $E_{1 k}(z)$ are two different combinations of the masking filters according to

$$
\begin{aligned}
& E_{0 k}(z)=\beta_{k} F_{0}^{(-k)}(z)+\beta_{k}^{*} F_{1}^{(+k)}(z), \\
& E_{1 k}(z)=\beta_{k}^{*} F_{0}^{(+k)}(z)+\beta_{k} F_{1}^{(-k)}(z) .
\end{aligned}
$$

The reason for this paraphrase is that the filters in (31) belong to Subclass I in [14] where useful formulas for ripple estimations are found. Using these formulas, as well as the fact that both $E_{0 k}(z)$ and $E_{1 k}(z)$ are the sum of the two filters $F_{0}(z)$ and $F_{1}(z)$, just shifted differently; the following restrictions on the different filters can be deduced:

$$
\begin{aligned}
& \delta_{c}^{\left(F_{0}\right)}+\delta_{s}^{\left(F_{1}\right)} \leq \min \left(\delta_{c}^{\left(E_{0}\right)}, \delta_{c}^{\left(E_{1}\right)}\right), \\
& \delta_{c}^{\left(F_{1}\right)}+\delta_{s}^{\left(F_{0}\right)} \leq \min \left(\delta_{c}^{\left(E_{0}\right)}, \delta_{c}^{\left(E_{1}\right)}\right), \\
& \delta_{s}^{\left(F_{0}\right)}+\delta_{s}^{\left(F_{1}\right)} \leq \min \left(\delta_{s}^{\left(E_{0}\right)}, \delta_{s}^{\left(E_{1}\right)}\right) .
\end{aligned}
$$

\footnotetext{
${ }^{3}$ The focus in this paper is on the design procedure, not the specific design criterion.
}

These formulas hold under the condition that second- and higher-order terms are neglected. As seen, $F_{0}(z)$ and $F_{1}(z)$ are restricted equally and we can use the simplified notations $\delta_{c}^{(F)}=\delta_{c}^{\left(F_{0}\right)}=\delta_{c}^{\left(F_{1}\right)}$ and $\delta_{s}^{(F)}=\delta_{s}^{\left(F_{0}\right)}=\delta_{s}^{\left(F_{1}\right)}$. Furthermore, $G(z)$ has the same ripples as its complementary filter, $\left[G_{c}(z)=G(-z)\right]$; thus $\delta_{c}^{(G)}=\delta_{c}^{\left(G_{c}\right)}$ and $\delta_{s}^{(G)}=\delta_{s}^{\left(G_{c}\right)}$. This implies that Case 1 and Case 2 with respect to the design do not differ, and the final simplified requirements on the subfilters regarding ripples are

$$
\begin{gathered}
\delta_{c}^{(F)}+\delta_{s}^{(F)}+\delta_{P C} \leq \delta_{c}, \\
\delta_{c}^{(F)}+\delta_{s}^{(F)}+\delta_{c}^{(G)} \leq \delta_{c}, \\
2\left(\delta_{s}^{(F)}\right)^{2}+\left(\delta_{s}^{(G)}\right)^{2} \leq \delta_{s}^{2}, \\
2 \delta_{s}^{(F)} \leq \delta_{s} .
\end{gathered}
$$

\subsection{Distortion function}

The distortion transfer function $V_{0}(z)$ is given by

$$
V_{0}(z)=\sum_{k=0}^{M-1} H_{a k}(z) H_{s k}(z) .
$$

In the appendix, it is shown that the frequency response of the distortion function can be expressed using the zero-phase frequency response $V_{0 R}(\omega T)$ as

$$
V_{0}\left(e^{j \omega T}\right)=e^{-j\left(N_{G} L+N_{F}\right) \omega T} V_{0 R}(\omega T),
$$

where

$$
\begin{aligned}
V_{0 R}(\omega T)=\sum_{k=0}^{M-1}( & {\left[G_{R}^{(-k)}(L \omega T)\right]^{2}\left[F_{0 R}^{(-k)}(\omega T)+F_{1 R}^{(+k)}(\omega T)\right]^{2} } \\
+ & {\left.\left[G_{c R}^{(-k)}(L \omega T)\right]^{2}\left[F_{0 R}^{(+k)}(\omega T)+F_{1 R}^{(-k)}(\omega T)\right]^{2}\right) }
\end{aligned}
$$

To have near PR, $V_{0}\left(e^{j \omega T}\right)$ should approximate a pure delay. Here, linear phase is fulfilled exactly (with a delay of $L N_{G}+N_{F}$ samples) and therefore it is enough to make sure that $V_{0 R}(\omega T)$ approximates one. Equation (36) leads to the following worst case ripple, ignoring second-order effects:

$$
2\left[\delta_{c}^{(F)}+\delta_{s}^{(F)}+\max \left(\delta_{P C}, \delta_{c}^{(G)}\right)\right] \leq \delta_{0} .
$$

\subsection{Aliasing functions}

Because of the decimation after the analysis filters in Figure 1, $M-1$ unwanted aliasing functions are introduced in the system. Their transfer functions are given in (24) for $m=$ $1, \ldots, M-1$ and should approximate zero in a near-PR FB. Normally in modulated FBs, adjacent terms in the aliasing functions are summed up to zero. This is called adjacentchannel aliasing cancellation [2]. By inserting the expressions for $H_{a k}(z)$ and $H_{s k}(z)$ as given by (7) and (8) into (23) and (24), we obtain expressions for all $V_{m}(z), m=1, \ldots, M-1$, and after a close investigation of these sums, the following 
conclusions can be drawn. There are two masking filters, but only the contribution from one of them (the largest overlap) is perfectly cancelled by adjacent-channel cancellation. Because of this, all the $M$ terms in each aliasing function will make a small contribution to the aliasing error. The maximal ripple is determined by the stopband ripple of the masking filters, $\delta_{s}^{(F)}$, and the squared stopband ripple of the model filter $\left(\delta_{s}^{(G)}\right)^{2}$. More precisely we get $5 \delta_{s}^{(F)}+2\left(\delta_{s}^{(G)}\right)^{2}$. Nonadjacent terms will have a maximum ripple of $2 \delta_{s}^{(F)}$ and we have $M-2$ of these terms. Therefore the worst case magnitude error for one aliasing function $\delta_{1}$ will be

$$
2(M-2) \delta_{s}^{(F)}+5 \delta_{s}^{(F)}+2\left(\delta_{s}^{(G)}\right)^{2} \leq \delta_{1} .
$$

For large $M$, this worst-case estimation of the aliasing functions will unfortunately be far from the real case. Therefore (38) is only useful for small and moderate values of $M$. A number of different filter banks have been synthesized, and these results indicate that $\delta_{1}$ typically have about the same size as $\delta_{0}$. This can be used as a guideline when designing filter banks for larger values of $M$.

\subsection{Estimation of optimal $L$}

The total number of multiplications per input/output sample (mults/sample) for the analysis (or synthesis) filter bank is expressed as

$$
R=2 \frac{N_{F}+1}{M}+\frac{N_{G}+1}{2},
$$

where $N_{G}$ is the filter order of $G(z)$ and $N_{F}$ is the filter order of $F_{0}(z)$ and $F_{1}(z)$. Both $N_{G}$ and $N_{F}$ depend on the periodicity factor $L$ in the FRM technique, and this implies that the arithmetic complexity is heavily dependent on the choice of $L$. Therefore, a formula is derived for estimating its optimal value. The filters $F_{0}(z)$ and $F_{1}(z)$ work at a sampling rate reduced by a factor $M$ and thereby their number of mults/sample is also decreased by the same factor. Further, $G(z)$ is symmetric and it is possible for its polyphase components $G_{0}(z)$ and $G_{1}(z)$ to share multipliers.

To estimate the filter order of an FIR filter, one can use the formula

$$
N=\frac{K}{\omega_{s} T-\omega_{c} T},
$$

where $\omega_{s} T$ and $\omega_{c} T$ are the stopband and passband edges of the filter. For $N_{F}$, a good approximation of $K$ is [8]

$$
K_{F}=2 \pi \frac{-20 \log \left(\sqrt{\delta_{s}^{(F)} \delta_{c}^{(F)}}\right)-13}{14.6}
$$

but for $N_{G}$, the additional condition of power complementarity [14] will increase the corresponding $K_{G}$. The masking filters $F_{0}(z)$ and $F_{1}(z)$ have the same transition bandwidth, $\pi / L-2 \Delta$, while the corresponding value for $G(z)$ is $2 L \Delta$. With (40) and (41) the total number of mults/sample can be estimated as

$$
R=\frac{2}{M}\left(\frac{K_{F}}{\pi / L-2 \Delta}+1\right)+\frac{1}{2}\left(\frac{K_{G}}{2 L \Delta}+1\right) .
$$

By finding the derivative of this expression with respect to $L$, the optimal $L$ can be found for each specification as ${ }^{4}$

$$
L_{\mathrm{opt}}=\frac{1}{(2 \Delta) / \pi+\sqrt{\left(8 \Delta K_{F}\right) /\left(M \pi K_{G}\right)}} .
$$

In addition, $L$ is restricted by the number of channels $M$, as $L=(4 m \pm 1) M$ in (5).

\section{DESIGN EXAMPLES}

To demonstrate the proposed design method, several modulated FBs are designed. ${ }^{5}$ In the first two examples, the specifications of and in (25)-(27) are the following: $\delta_{c}=\delta_{s}=$ $\delta_{0}=\delta_{1}=0.01$. Further, the number of channels $M$ varies and determines the width of the transition band $2 \Delta$, with $\Delta=0.025 \pi / M$. The third example is a comparison to [18, Example 2]. The interesting aspect to study when comparing multirate FBs is not the filter orders, but the number of multiplications per input/output sample (number of multiplications at the lower rate), here denoted as mults/sample. This is because different filters can work at different sample rates. For the proposed FBs, the number of mults/sample can be calculated as in (39), whereas with a regular FIR prototype filter of order $N$, it is simply $2((N+1) / M)$. One should also keep in mind that the modulation blocks also contribute to the total arithmetic complexity of the FBs and that only one is needed with a regular FIR prototype filter or with the approach in [18]. This contribution is however independent of the filter orders and has a relatively low complexity compared to the filter part. It is therefore not discussed here.

Example 1. A FB with $M=5$ was designed and the estimated optimal $L$ was found to be either 5 or 15 , depending on the choice of $K_{G}$ in Section 4.4. Both cases were considered, and 15 was found to give the FB with lowest complexity for the given specification. Translating the specification to restrictions on the three subfilters gives $\delta_{c}^{(F)}=0.001, \delta_{s}^{(F)}=$ $0.00085, \delta_{c}^{(G)}=0.0031, \delta_{P C}=0.0031$, and $\delta_{s}^{(G)}=0.0099$. These specifications are met with filter orders $N_{G}=47$ and $N_{F}=114$. Further, with successive decrement of $N_{F}$, the specification was found to be fulfilled for $N_{F} \geq 102$. Magnitude responses of the analysis filters, distortion function, and aliasing functions with $N_{F}=102$ are plotted in Figures 7, 8, and 9. Using nonlinear optimization, the filter orders could be lowered to $N_{G}=39$ and $N_{F}=58$ and still meet the specification. This shows that for this particular specification, there was a large design margin. The corresponding magnitude responses are depicted in Figures 10, 11, and 12. Using (39), the implementation cost without the nonlinear optimization procedure for the overall FB (including the

\footnotetext{
${ }^{4}$ The variable $K_{G}$ is assumed to be independent of $L$.

${ }^{5}$ For the joint optimization, the Matlab function fminimax.m has been used.
} 


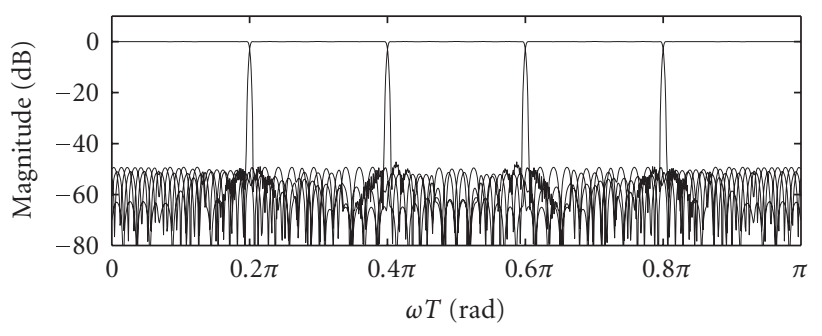

FIGURE 7: Magnitude responses of the analysis filters without the nonlinear optimization procedure with $N_{G}=47$ and $N_{F}=102$, Example 1.

analysis and synthesis parts) is 130.4 mults/sample plus the cost to implement the cosine and sine modulation blocks. After the nonlinear optimization procedure, the number is only 87.2.

As a comparison, the estimated complexity of a regular FIR $^{6}$ cosine modulated NPR FB would need a filter order of about 580. Therefore, at least about 232 mults/sample are needed in the filter part using a regular FIR prototype filter. Thus, even without the nonlinear optimization procedure, the proposed method gives a solution with substantially lower arithmetic complexity.

As usual when employing the FRM technique, we achieve more savings when the transition band becomes more narrow. The price to pay for the decreased arithmetic complexity and the decreased number of optimization parameters is, as always when using an FRM approach with linear-phase subfilters, a longer overall delay. In this example, the delay is about 39\% longer for the proposed FB without joint optimization compared to the regular FB. With joint optimization, the figure is decreased to $11 \%$.

Example 2. With increasing $M$, also $L$ increases and it becomes difficult to optimize the different filters together in the minimax sense. However, optimizing them separately, also gives good results. Filter banks with $M=8,16,32$, and 256 were designed, and the optimal $L$ was found to be 24,48 , 96 , and 768, respectively. The number of multiplications required per sample in the filter parts is visualized in Table 1. For comparison reasons, the estimated complexity with a regular FIR prototype filter (estimated as above) is also given. Further, the total delay of the filter parts of the different FBs is given, as well as the number of distinct filter coefficients to optimize. When the number of channels is doubled, the transition bands of the masking filters and the regular FIR filter are halved. This corresponds to an approximately doubled filter order. But since the sampling rate for the filters is also halved, the number of multiplications per sample remains about the same. This is the reason for the limited variations for different $M$ in Table 1 . For further illustration,

\footnotetext{
6 The estimation is taken from the 2-channel case, and then when generalizing, the filter order is assumed to be proportional to the transition bandwidth.
}

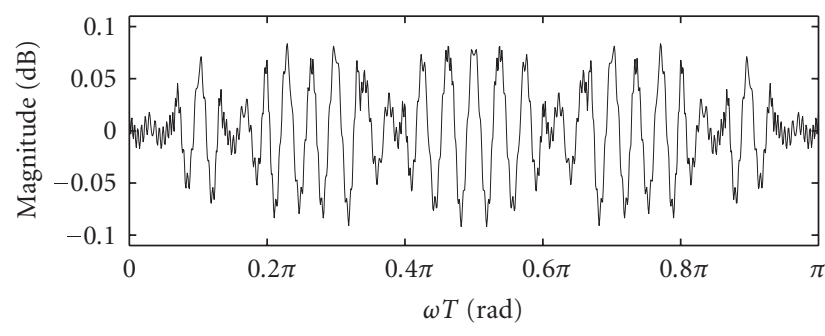

FIGURE 8: Magnitude response of the distortion function without the nonlinear optimization procedure with $N_{G}=47$ and $N_{F}=102$, Example 1.

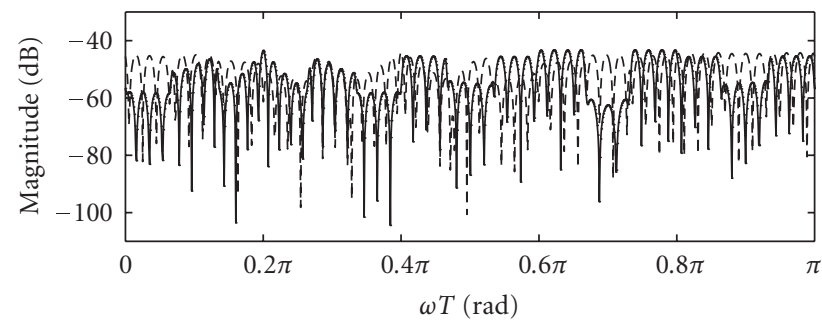

FIGURE 9: Magnitude responses of the aliasing functions without the nonlinear optimization procedure with $N_{G}=47$ and $N_{F}=102$, Example 1.

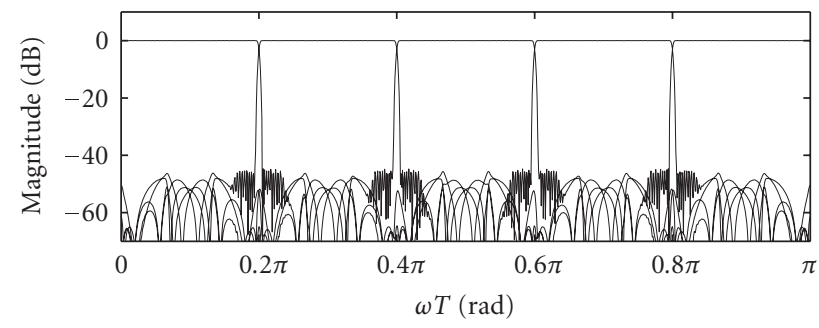

FIGURE 10: Magnitude responses of the analysis filters with $N_{G}=39$ and $N_{F}=58$, Example 1 .

some details for $M=32$ are given. When (33) and (37) are used to distribute the ripples ( $(38)$ is not considered because of the size of $M$ ), the required filter orders were $N_{G}=47$ and $N_{F}=716$. With a successive decrement of $N_{F}$, the specification was found to be fulfilled for $N_{F} \geq 658 .^{7}$ The ripples after the separate design are $\delta_{c}<0.0040, \delta_{s}<0.0034, \delta_{0}<0.0096$, and $\delta_{1}<0.0071$, and the magnitude response of the analysis filters is shown in Figure 13.

Example 3. A comparison with [18, Example 2] has been made and the results are summarized in Table 2 . The data in the first column is synthesized with $L=24$. The second column corresponds to a separate design of the subfilters us-

\footnotetext{
7 The decrease of $N_{F}$ may seem large, but it only corresponds to a reduction of $5 \%$ of the overall complexity.
} 


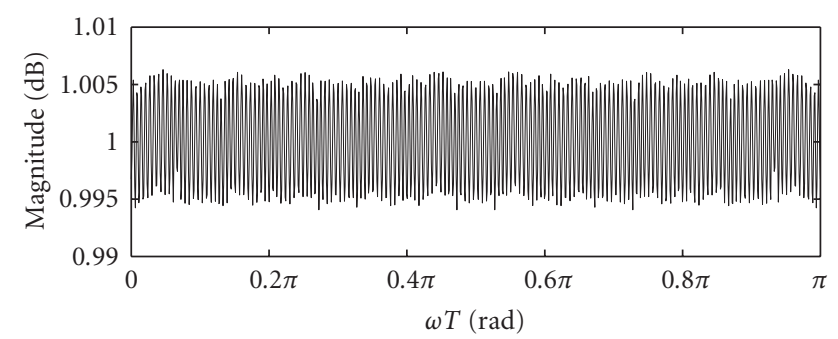

Figure 11: Magnitude response of the distortion function without the nonlinear optimization procedure with $N_{G}=39$ and $N_{F}=58$, Example 1.

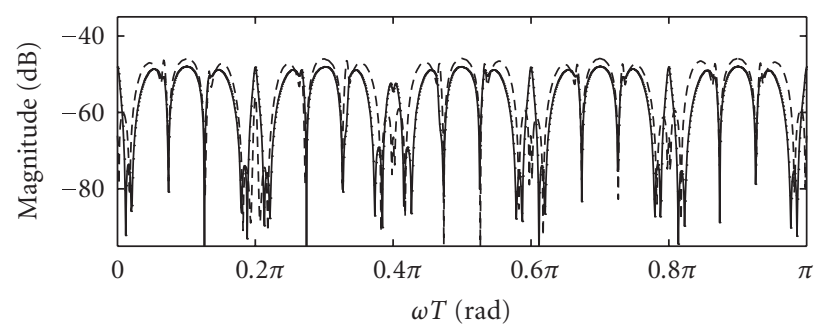

FIGURE 12: Magnitude responses of the aliasing functions without the nonlinear optimization procedure with $N_{G}=39$ and $N_{F}=58$, Example 1.

TABle 1: Number of multiplications per sample, total delay, and number of optimization parameters using the proposed prototype filters or a regular FIR prototype filter, for different numbers of channels.

\begin{tabular}{lrccr}
\hline FB class & $M$ & Mults/sample & Coefficients & Delay \\
\hline Proposed & 8 & 130.5 & 190 & 1292 \\
Regular FIR & 8 & 232.25 & 465 & 928 \\
Proposed & 16 & 129.75 & 352 & 2582 \\
Regular FIR & 16 & 232.125 & 929 & 1856 \\
Proposed & 32 & 130.375 & 683 & 5170 \\
Regular FIR & 32 & 232.0625 & 1857 & 3712 \\
Proposed & 256 & 132.39 & 5426 & 41496 \\
Regular FIR & 256 & 232.008 & 14849 & 29696 \\
\hline
\end{tabular}

ing the distribution formulas given in (33), (37), and (38), with $L=24$. In the last column, results with $L=40$ are presented. When the distribution formulas for $L=40$ were used, $N_{F 0}$ and $N_{F 1}$ were found to be 361 , but after the separate optimization, it was possible to lower these orders to $329 .{ }^{8} \mathrm{No}$ joint optimization has been performed on the FBs in column two or three; thus these results can be improved further.

In terms of distinct coefficients, $L=24$ is the best choice, but if the number of mults/sample is more interesting, the

\footnotetext{
${ }^{8}$ For $L=24$, it was not possible to decrease the filter orders.
}

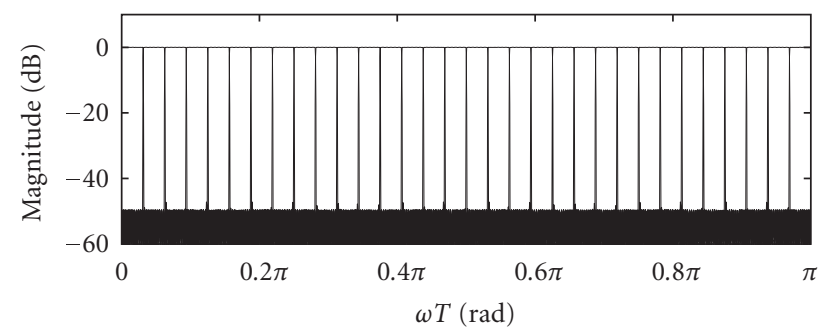

FIGURE 13: Magnitude responses of the analysis filters with separate optimization for $M=32$, Example 2 .

TABle 2: Comparison with [18, Example 2].

\begin{tabular}{lccc}
\hline & [18, Example 2] & $L=24$ & $L=40$ \\
\hline$N_{G}$ & 186 & 169 & 101 \\
$N_{F 0}\left(N_{F 1}\right)$ & 143 & 210 & 329 \\
$\delta_{s}$ & 0.0014 & 0.0014 & 0.0014 \\
$\delta_{0}$ & 0.009 & 0.00047 & 0.006 \\
$\delta_{1}$ & 0.0018 & 0.00051 & 0.00081 \\
Coefficients & $475(238)$ & 297 & 383 \\
Mults./sample & 446 & 275.5 & 267 \\
Delay & 4607 & 4266 & 4369 \\
\hline
\end{tabular}

solution with $L=40$ is preferable. Due to the extra upsamplers in [18], some subfilters work at a higher sampling rate compared to our proposal. This seems to be the main explanation to the significant difference (40\% decrease) in arithmetic complexity. The number of distinct coefficients to be optimized given in [18, Example 2] is 475 , but since their three subfilters all have linear phase, the correct number seems more likely to be 238 . However, using the number given in the example, the proposed FBs have about 20\% less optimization parameters.

\section{CONCLUSION}

This paper introduced an approach for synthesizing modulated maximally decimated FIR FBs using the FRM technique. For this purpose, a new class of FRM filters was introduced. Each of the analysis and synthesis FBs is realized with the aid of three filters, one cosine modulation block, and one sine modulation block. The overall FBs achieve nearly PR with a linear-phase distortion function. Further, a design procedure is given, allowing synthesis of a general FB specification. Compared to similar approaches, the proposed FBs have about $40 \%$ lower arithmetic complexity. Compared to regular cosine modulated FIR FBs, both the overall arithmetic complexity and the number of distinct filter coefficients are significantly reduced, at the expense of an increased overall FB delay in applications requiring narrow transition bands. These statements were demonstrated by means of several design examples. 


\section{APPENDIX}

This appendix shows some of the properties of the proposed FBs concerning the prototype filters, the analysis filters, and the synthesis filters.

We first regard the magnitude response of the prototype filters and the phase response of $P_{a}\left(e^{j \omega T}\right) P_{s}\left(e^{j \omega T}\right)$ (properties (1) and (2) in Section 3.3). The frequency responses of $G\left(e^{j \omega T}\right), G_{c}\left(e^{j \omega T}\right), F_{0}\left(e^{j \omega T}\right)$, and $F_{1}\left(e^{j \omega T}\right)$ can be written as

$$
\begin{aligned}
& G\left(e^{j \omega T}\right)=e^{-j N_{G} \omega T / 2} G_{R}(\omega T), \\
& G_{c}\left(e^{j \omega T}\right)=e^{-j N_{G} \omega T / 2} G_{c R}(\omega T), \\
& F_{0}\left(e^{j \omega T}\right)=e^{-j N_{F} \omega T / 2} F_{0 R}(\omega T), \\
& F_{1}\left(e^{j \omega T}\right)=e^{-j N_{F} \omega T / 2} F_{1 R}(\omega T),
\end{aligned}
$$

where $G_{R}(\omega T), G_{c R}(\omega T), F_{0 R}(\omega T)$, and $F_{1 R}(\omega T)$ denote zero-phase frequency responses. We rewrite the magnitude responses of the prototype filters in (2) and (3) as

$$
\begin{aligned}
& P_{a}\left(e^{j \omega T}\right) \\
& \quad=G\left(e^{j L \omega T}\right) F_{0}\left(e^{j \omega T}\right)+G_{c}\left(e^{j L \omega T}\right) F_{1}\left(e^{j \omega T}\right) \\
& \quad=e^{-j\left(N_{G} L+N_{F}\right) \omega T / 2}\left[G_{R}(L \omega T) F_{0 R}(\omega T)+j G_{c R}(L \omega T) F_{1 R}(\omega T)\right], \\
& P_{S}\left(e^{j \omega T}\right) \\
& \quad=G\left(e^{j L \omega T}\right) F_{0}\left(e^{j \omega T}\right)-G_{c}\left(e^{j L \omega T}\right) F_{1}\left(e^{j \omega T}\right) \\
& \quad=e^{-j\left(N_{G} L+N_{F}\right) \omega T / 2}\left[G_{R}(L \omega T) F_{0 R}(\omega T)-j G_{c R}(L \omega T) F_{1 R}(\omega T)\right] .
\end{aligned}
$$

From (A.2) it follows that the squared magnitude response of the two prototype filters are

$$
\begin{aligned}
\left|P_{a}\left(e^{j \omega T}\right)\right|^{2} & =G_{R}^{2}(L \omega T) F_{0 R}^{2}(\omega T)+G_{c R}^{2}(L \omega T) F_{1 R}^{2}(\omega T) \\
& =\left|P_{s}\left(e^{j \omega T}\right)\right|^{2}
\end{aligned}
$$

thus identical. Further, the product of the two magnitude responses has linear phase, as can be seen in (A.4) below. Hereafter, $(\omega T)$ and $(L \omega T)$ are left out for the sake of simplicity,

$$
\begin{aligned}
P_{a}\left(e^{j \omega T}\right) P_{s}\left(e^{j \omega T}\right)= & e^{-j\left(N_{G} L+N_{F}\right) \omega T}\left(G_{R} F_{0 R}+j G_{c R} F_{1 R}\right) \\
& \cdot\left(G_{R} F_{0 R}-j G_{c R} F_{1 R}\right) \\
= & e^{-j\left(N_{G} L+N_{F}\right) \omega T}\left(G_{R}^{2} F_{0 R}^{2}+G_{c R}^{2} F_{1 R}^{2}\right) .
\end{aligned}
$$

Secondly, we show that the magnitude responses of the analysis filters and the synthesis filters are equal, and that the product of $H_{a k}\left(e^{j \omega T}\right)$ and $H_{s k}\left(e^{j \omega T}\right)$ has a linear-phase response with delay $L N_{G}+N_{F}$ (properties (3) and (4) in Section 3.3). We use the notation in (29) and rewrite the transfer functions of the analysis filters, (7), and the synthesis filters, (8), as

$$
\begin{aligned}
& H_{a k}(z)=\beta_{k} P_{a}^{(-k)}(z)+\beta_{k}^{*} P_{s}^{(+k)}(z) \\
& =\beta_{k}\left[G^{(-k)}\left(z^{L}\right) F_{0}^{(-k)}(z)+G_{c}^{(-k)}\left(z^{L}\right) F_{1}^{(-k)}(z)\right] \\
& +\beta_{k}^{*}\left[G^{(+k)}\left(z^{L}\right) F_{0}^{(+k)}(z)+G_{c}^{(+k)}\left(z^{L}\right) F_{1}^{(+k)}(z)\right], \\
& =c j\left(-1^{k}\right)\left[\beta_{k} P_{s}^{(-k)}(z)-\beta_{k}^{*} P_{s}^{(+k)}(z)\right] \\
& =c j\left(-1^{k}\right)\left[\beta_{k}\left(G^{(-k)}\left(z^{L}\right) F_{0}^{(-k)}(z)-G_{c}^{(-k)}\left(z^{L}\right) F_{1}^{(-k)}(z)\right)\right. \\
& \left.+\beta_{k}^{*}\left(G^{(+k)}\left(z^{L}\right) F_{0}^{(+k)}(z)-G_{c}^{(+k)}\left(z^{L}\right) F_{1}^{(+k)}(z)\right)\right] .
\end{aligned}
$$

We use the fact that

$$
\begin{gathered}
\left(e^{ \pm j((2 k+1) / 2 M) \pi} z\right)^{2 L}=-z^{2 L}, \\
\left(e^{j((2 k+1) / 2 M) \pi} z\right)^{L}= \pm j(-1)^{k} z^{L}, \\
\left(e^{-j((2 k+1) / 2 M) \pi} z\right)^{L}=\mp j(-1)^{k} z^{L},
\end{gathered}
$$

where the plus or minus sign depends on $k$ and on $m$ in (5). Rewriting the model filters using their polyphase components we get

$$
\begin{aligned}
& G^{(-k)}\left(z^{L}\right)=G_{0}\left(-z^{2 L}\right) \mp j(-1)^{k} z^{-L} G_{1}\left(z^{2 L}\right), \\
& G^{(+k)}\left(z^{L}\right)=G_{0}\left(-z^{2 L}\right) \pm j(-1)^{k} z^{-L} G_{1}\left(z^{2 L}\right), \\
& G_{c}^{(-k)}\left(z^{L}\right)=G_{0}\left(-z^{2 L}\right) \pm j(-1)^{k} z^{-L} G_{1}\left(z^{2 L}\right), \\
& G_{c}^{(+k)}\left(z^{L}\right)=G_{0}\left(-z^{2 L}\right) \mp j(-1)^{k} z^{-L} G_{1}\left(z^{2 L}\right) .
\end{aligned}
$$

This gives us the following relation between $G(z)$ and $G_{c}(z)$ :

$$
G^{(-k)}\left(z^{L}\right)=G_{c}^{(+k)}\left(z^{L}\right), \quad G^{(+k)}\left(z^{L}\right)=G_{c}^{(-k)}\left(z^{L}\right) .
$$

Now we rewrite the transfer function of the analysis and synthesis filters as

$$
\begin{aligned}
& H_{a k}(z)= G^{(-k)}\left(z^{L}\right)\left[\beta_{k} F_{0}^{(-k)}(z)+\beta_{k}^{*} F_{1}^{(+k)}(z)\right] \\
&+G_{c}^{(-k)}\left(z^{L}\right)\left[\beta_{k}^{*} F_{0}^{(+k)}(z)+\beta_{k} F_{1}^{(-k)}(z)\right], \\
& H_{s k}(z)=c j(-1)^{k}\left(G^{(-k)}\left(z^{L}\right)\left[\beta_{k} F_{0}^{(-k)}(z)+\beta_{k}^{*} F_{1}^{(+k)}(z)\right]\right. \\
&\left.\quad-G_{c}^{(-k)}\left(z^{L}\right)\left[\beta_{k}^{*} F_{0}^{(+k)}(z)+\beta_{k} F_{1}^{(-k)}(z)\right]\right) .
\end{aligned}
$$


We use (A.9) and omit $(\omega T)$ and $(L \omega T)$ to write their frequency responses as

$$
\begin{aligned}
H_{a k}\left(e^{j \omega T}\right)= & e^{-j / 2\left(N_{G} L+N_{F}\right) \omega T \mp j(k+0.5) \pi N_{G}} \\
& \cdot\left[G_{R}^{(-k)}\left(F_{0 R}^{(-k)}+F_{1 R}^{(+k)}\right)+j G_{c R}^{(-k)}\left(F_{0 R}^{(+k)}+F_{1 R}^{(-k)}\right)\right], \\
H_{s k}\left(e^{j \omega T}\right)= & c j(-1)^{k} e^{-j / 2\left(N_{G} L+N_{F}\right) \omega T \mp j(k+0.5) \pi N_{G}} \\
& \cdot\left[G_{R}^{(-k)}\left(F_{0 R}^{(-k)}+F_{1 R}^{(+k)}\right)-j G_{c R}^{(-k)}\left(F_{0 R}^{(+k)}+F_{1 R}^{(-k)}\right)\right] .
\end{aligned}
$$

From this, it follows that the magnitude of the frequency responses are equal, as can be seen in (A.11) below,

$$
\begin{aligned}
& \left|H_{a k}\left(e^{j \omega T}\right)\right|=\left|G_{R}^{(-k)}\left(F_{0 R}^{(-k)}+F_{1 R}^{(+k)}\right)+j G_{c R}^{(-k)}\left(F_{0 R}^{(+k)}+F_{1 R}^{(-k)}\right)\right|, \\
& \left|H_{s k}\left(e^{j \omega T}\right)\right|=\left|G_{R}^{(-k)}\left(F_{0 R}^{(-k)}+F_{1 R}^{(+k)}\right)-j G_{c R}^{(-k)}\left(F_{0 R}^{(+k)}+F_{1 R}^{(-k)}\right)\right| .
\end{aligned}
$$

Finally, since $e^{\mp j(k+0.5) \pi N_{G}}=-c j(-1)^{k}$, the product of the filters $H_{a k}\left(e^{j \omega T}\right)$ and $H_{s k}\left(e^{j \omega T}\right)$ is

$$
\begin{aligned}
& H_{a k}\left(e^{j \omega T}\right) H_{s k}\left(e^{j \omega T}\right) \\
& =e^{-j\left(N_{G}+N_{F}\right) \omega T} \cdot\left[\left(G_{R}^{(-k)}\right)^{2}\left(F_{0 R}^{(-k)}+F_{1 R}^{(+k)}\right)^{2}\right. \\
& \left.+\left(G_{c R}^{(-k)}\right)^{2}\left(F_{0 R}^{(+k)}+F_{1 R}^{(-k)}\right)^{2}\right]
\end{aligned}
$$

and thus

$$
\begin{aligned}
V_{0}\left(e^{j \omega T}\right)= & \sum_{k=0}^{M-1} H_{a k}\left(e^{j \omega q T}\right) H_{s k}\left(e^{j \omega T}\right) \\
= & \sum_{k=0}^{M-1}\left(e ^ { - j ( N _ { G } + N _ { F } ) \omega T } \left[\left(G_{R}^{(-k)}\right)^{2}\left(F_{0 R}^{(-k)}+F_{1 R}^{(+k)}\right)^{2}\right.\right. \\
& \left.\left.+\left(G_{c R}^{(-k)}\right)^{2}\left(F_{0 R}^{(+k)}+F_{1 R}^{(-k)}\right)^{2}\right]\right)
\end{aligned}
$$

which obviously has a linear-phase response of $-\left(N_{G} L+\right.$ $\left.N_{F}\right) \omega T$.

\section{REFERENCES}

[1] R. E. Crochiere and L. R. Rabiner, Multirate Digital Signal Processing, Prentice-Hall, Englewood Cliffs, NJ, USA, 1983.

[2] P. P. Vaidyanathan, Multirate Systems and Filter Banks, Prentice-Hall, Englewood Cliffs, NJ, USA, 1993.

[3] N. J. Fliege, Multirate Digital Signal Processing, John Wiley \& Sons, New York, NY, USA, 1994.

[4] T. Saramäki, "A generalized class of cosine modulated filter banks," in Proceedings of the 1st International Workshop on Transforms and Filter Banks, pp. 336-365, Tampere, Finland, February 1998.

[5] R. Bregović and T. Saramäki, "An efficient approach for designing nearly perfect-reconstruction low-delay cosinemodulated filter banks," in Proceedings of the IEEE International Symposium on Circuits and Systems, vol. 1, pp. 825-828, Phoenix, Ariz, USA, May 2002.
[6] L. Svensson, P. Löwenborg, and H. Johansson, "A class of cosine-modulated causal IIR filter banks," in Proceedings of the 9th International Conference on Electronics, Circuits and Systems (ICECS '02), vol. 3, pp. 915-918, Dubrovnik, Croatia, September 2002.

[7] A. Eshraghi and T. S. Fiez, "A comparative analysis of parallel delta-sigma ADC architectures," IEEE Transactions on Circuits and Systems I: Regular Papers, vol. 51, no. 3, pp. 450-458, 2004.

[8] J. F. Kaiser, "Nonrecursive digital filter design using $I_{0}$-sinh window function," in Proceedings of the IEEE Symposium on Circuits \& Systems (ISCAS '74), vol. 3, pp. 20-23, San Francisco, Calif, USA, April 1974.

[9] T. Saramäki, "Finite impulse response filter design," in Handbook for Digital Signal Processing, S. K. Mitra and J. F. Kaiser, Eds., chapter 4, pp. 155-277, John Wiley \& Sons, New York, NY, USA, 1993.

[10] Y. C. Lim, "Frequency-response masking approach for the synthesis of sharp linear phase digital filters," IEEE Transactions on Circuits and Systems, vol. 33, no. 4, pp. 357-364, 1986.

[11] Y. C. Lim and Y. Lian, "The optimum design of one and twodimensional FIR filters using the frequency response masking technique," IEEE Transactions on Circuits and Systems II: Analog and Digital Signal Processing, vol. 40, no. 2, pp. 88-95, 1993.

[12] T. Saramäki, "Design of computationally efficient FIR filters using periodic subfilters as building blocks," in The Circuits and Filters Handbook, W. K. Chen, Ed., pp. 2578-2601, CRC Press, Boca Raton, Fla, USA, 1995.

[13] H. Johansson and T. Saramäki, "Two-channel FIR filter banks based on the frequency-response masking approach," in Proceedings of the 2nd International Workshop on Transforms Filter Banks, Brandenburg an der Havel, Germany, March 1999.

[14] H. Johansson, "New classes of frequency-response masking FIR filters," in Proceedings of the IEEE International Symposium on Circuits and Systems, vol. 3, pp. 81-84, Geneva, Switzerland, May 2000.

[15] P. S. R. Diniz, L. C. R. De Barcellos, and S. L. Netto, "Design of cosine-modulated filter bank prototype filters using the frequency-response masking approach," in Proceedings of the IEEE International Conference on Acoustics, Speech and Signal Processing (ICASSP '01), vol. 6, pp. 3621-3624, Salt Lake, Utah, USA, May 2001.

[16] M. B. Furtado Jr., P. S. R. Diniz, and S. L. Netto, "Optimized prototype filter based on the FRM approach for cosinemodulated filter banks," Circuits, Systems, and Signal Processing, vol. 22, no. 2, pp. 193-210, 2003.

[17] S. L. Netto, L. C. R. De Barcellos, and P. S. R. Diniz, "Efficient design of narrowband cosine-modulated filter banks using a two-stage frequency-response masking approach," Journal of Circuits, Systems and Computers, vol. 12, no. 5, pp. 631-642, 2003.

[18] P. S. R. Diniz, L. C. R. De Barcellos, and S. L. Netto, "Design of high-resolution cosine-modulated transmultiplexers with sharp transition band," IEEE Transactions on Signal Processing, vol. 52, no. 5, pp. 1278-1288, 2004.

[19] M. B. Furtado Jr., P. S. R. Diniz, S. L. Netto, and T. Saramäki, "On the design of high-complexity cosine-modulated transmultiplexers based on the frequency-response masking approach," IEEE Transactions on Circuits and Systems I: Regular Papers, vol. 52, no. 11, pp. 2413-2426, 2005.

[20] L. Svensson, P. Löwenborg, and H. Johansson, "Modulated $m$-channel FIR filter banks utilizing the frequency response masking approach," in Proceedings of the IEEE Nordic Signal Processing Symposium (NORSIG '02), Hurtigruta, TromsöTrondheim, Norway, October 2002. 
[21] L. Rosenbaum, P. Löwenborg, and H. Johansson, "Cosine and sine modulated FIR filter banks utilizing the frequencyresponse masking approach," in Proceedings of the IEEE International Symposium on Circuits and Systems, vol. 3, pp. 882885, Bangkok, Thailand, May 2003.

[22] J. H. McClellan, T. W. Parks, and L. R. Rabiner, "A computer program for designing optimum FIR linear phase digital filters," IEEE Transactions on Audio and Electroacoustics, vol. 21, no. 6, pp. 506-526, 1973.

Linnéa Rosenbaum (maiden name Svensson) was born in Färgaryd, Sweden, in 1976. She received the M.S. degree in applied physics and electrical engineering and the Licentiate degree in electronics systems from Linköping University, Sweden, in 2001 and 2003 , respectively. She is currently pursuing her studies for the Doctoral degree. Her research interests are digital filters with emphasis on realization and implementa-

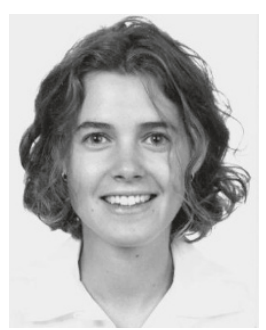
tion of filter banks. She received the IEEE Nordic Signal Processing Symposium Best Paper Award 2002.

Per Löwenborg was born in Oskarshamn, Sweden, in 1974. He received the M.S. degree in applied physics and electrical engineering and the Licentiate and Doctoral degrees in electronics systems from Linköping University, Sweden, in 1998, 2001, and 2002, respectively. His research interests are within the field of theory, design, and implementation of analog and digital signal processing electronics. He is the author or

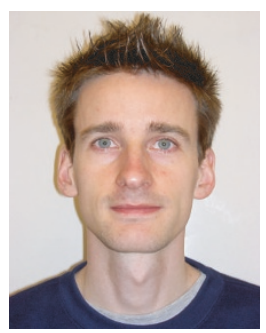
coauthor of one book and more than 50 international journals and conference papers. He was awarded the 1999 IEEE Midwest Symposium on Circuits and Systems Best Student Paper Award and the 2002 IEEE Nordic Signal Processing Symposium Best Paper Award. He is a Member of the IEEE.

Håkan Johansson was born in Kumla, Sweden, in 1969. He received the M.S. degree in computer science and the Licentiate, Doctoral, and Docent degrees in electronics systems from Linköping University, Sweden, in 1995, 1997, 1998, and 2001, respectively. During 1998 and 1999, he held a postdoctoral position at Signal Processing Laboratory, Tampere University of Technology, Finland. He is currently a Professor in elec-

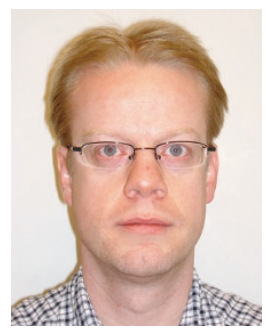
tronics systems at the Department of Electrical Engineering of Linköping University. His research interests include theory, design, and implementation of signal processing systems. He is the author or coauthor of four textbooks and more than 100 international journals and conference papers. He has served/serves as an Associate Editor for the IEEE Transactions on Circuits and SystemsII (2000-2001), IEEE Signal Processing Letters (2004-2007), and IEEE Transactions on Signal Processing (2006-2008), and he is a Member of the IEEE International Symposium on Circuits and Systems DSP Track Committee. 